E. Freitag and M. Oura

Nagoya Math. J.

Vol. 161 (2001), 69-83

\title{
A THETA RELATION IN GENUS 4
}

\author{
EBERHARD FREITAG AND MANABU OURA ${ }^{1}$
}

\begin{abstract}
The $2^{g}$ theta constants of second kind of genus $g$ generate a graded ring of dimension $g(g+1) / 2$. In the case $g \geq 3$ there must exist algebraic relations. In genus $g=3$ it is known that there is one defining relation. In this paper we give a relation in the case $g=4$. It is of degree 24 and has the remarkable property that it is invariant under the full Siegel modular group and whose $\Phi$-image is not zero. Our relation is obtained as a linear combination of code polynomials of the 9 self-dual doubly-even codes of length 24 .
\end{abstract}

\section{Introduction}

The theta constants of genus $g$ of second kind are the $2^{g}$ functions

$$
f_{a}(Z):=\sum_{n \in \mathbb{Z}^{g}} \exp 2 \pi \mathrm{i} Z[n+a / 2] .
$$

They generate a ring of dimension $g(g+1) / 2+1$. In the cases $g \leq 2$ they are algebraically independent, in the case $g=3$ there is a defining relation in degree 16, which is related to the so-called Schottky relation [Ru3]. In this paper we describe a similar relation in genus 4 . Its degree is 24 . This relation has two remarkable properties. It is invariant under the full Siegel modular group and it is mapped to a non trivial relation under Siegel $\Phi$-operator.

It is a natural question, whether our relation is a consequence of Riemann's theta relations. We could not decide this. One reason is that the Riemann theta relations are quartic relations in the theta constants of first kind

$$
\vartheta[m]=\sum_{n \in \mathbb{Z}^{g}} \exp \pi \mathrm{i}\left(Z[n+a / 2]+b^{\prime}(n+a / 2)\right), \quad m=\left(\begin{array}{l}
a \\
b
\end{array}\right) \in \mathbb{Z}^{2 g} .
$$

The functions $f_{a} f_{b}$ generate the same vetor space as the squares $\vartheta[m]^{2}$. We learnt from B. van Geemen and R. Salvati-Manni how to obtain relations

Received April 24, 1999.

1991 Mathematics Subject Classification: 11T71 94B05 11F46 11F27.

${ }^{1}$ The second author's work was supported in part by a grant from the Japan Society for the Promotion of Science. 
between the $f_{a}$ from the Riemann relations using elimination tricks. But it seems very hard to obtain the distinguished relation which we describe below.

Our relation gives rise to a certain Siegel cusp form of weight 12 and genus 5 with respect to the full Siegel modular group.

We use the theory of codes. There are 9 isomorphy classes of binary selfdual doubly-even codes in $\mathbb{F}_{2}^{24}$. They are associated to 9 of the 24 Niemeier lattices. To any binary code $C$ and any genus $g$ there is associated a certain code polynomial, which is a polynomial in $2^{g}$ variables $F_{a}, a \in \mathbb{F}_{2}^{g}$. If one replaces $F_{a}$ by $f_{a}$ one obtains a function which is nothing else but the usual theta function of the lattice $L$ which is related to the code $C$. Recall that $L$ is the inverse image of $C \subset \mathbb{F}_{2}^{n}$ in $\mathbb{Z}^{n}$ with the scalar product $2\langle x, y\rangle=$ $\sum x_{i} y_{i}$. Our relation will be constructed as a linear combination of the code polynomials of the nine mentioned codes.

With help of computers we determined completely the code polynomials of genus 4 of the nine "Niemeier codes". The tables are printed at the end of this paper.

Section 1 of the paper contains the results of the paper. In Section 2 some of the computational problems are explained. Tables can be found in Section 3.

We would like to thank R. Salvati-Manni, B. van Geemen and B. Runge for helpful discussions. This work was done during the second author's stay in Mannheim. He would like to thank Professor R. Weissauer for his hospitality.

\section{$\S 1$. Siegel modular forms of genus four and codes}

Let $\mathbb{F}_{2}$ be the field of two elements and $V=\mathbb{F}_{2}^{g}$. Sometimes we will identify the elements of $\mathbb{F}_{2}$ with the integers 0 , 1. For $a, b \in V$ let $a \cdot b$ denote the standard scalar product. Set

$$
T_{g}:=\left(\frac{1+\mathrm{i}}{2}\right)^{g}\left((-1)^{a \cdot b}\right)_{a, b \in V}
$$

and for any symmetric $g \times g$-matrix with integral coefficients

$$
D_{S}:=\operatorname{diag}\left(\mathrm{i}^{S[a]} \text { with } a \in V\right)
$$

where

$$
S[a]:=\sum_{i, j} s_{i j} a_{i} a_{j} \quad(\in \mathbb{Z}) .
$$


The group generated by the matrices $T_{g}$ and $D_{S}$ is

$$
H_{g}:=\left\langle T_{g}, D_{S}\right\rangle .
$$

After a choice of an ordering of $V$ this is a subgroup of $\mathrm{GL}\left(2^{g}, \mathbb{C}\right)$. It is known that $H_{g}$ is a finite group. We introduce for every $a \in V$ a variable $F_{a}$ and consider the ring of invariants

$$
R_{g}:=\mathbb{C}\left[\left(F_{a}\right)_{a \in V}\right]^{H_{g}} .
$$

This ring is connected with the ring $A\left(\Gamma_{g}\right)$ of Siegel modular forms. The link comes from the "theta constants of second kind":

$$
f_{a}(Z):=\sum_{n \in \mathbb{Z}^{g}} \exp 2 \pi \mathrm{i} Z[n+a / 2] .
$$

Here $Z$ varies in the Siegel half plane of genus $g$ which consists of all symmetric $g \times g$-matrices with positive definite imaginary part. From the classical theta transformation formula one knows that the evaluation $F_{a} \mapsto f_{a}$ defines a homomorphism

$$
R_{g} \longrightarrow A_{g}
$$

Here

$$
A_{g}=A\left(\Gamma_{g}\right)=\sum_{k=0}^{\infty}\left[\Gamma_{g}, k\right]
$$

where $\left[\Gamma_{g}, k\right]$ denotes the vector space of Siegel modular forms of weight $k$ (with respect to the full Siegel modular group $\Gamma_{g}=\operatorname{Sp}(g, \mathbb{Z})$ ). We use the weight convention that a form of weight $k$ transforms as

$$
f\left((A Z+B)(C Z+D)^{-1}\right)=\operatorname{det}(C Z+D)^{k} f(Z) .
$$

The weight of the $f_{a}$ have to be counted as $1 / 2$.

A variant of "Igusa's fundamental lemma" states that the subring
$A_{g}^{(2)} \subset A_{g}$ of modular forms of even weights equals the normalization of the image of the ring $R_{g}$. From this point of view the determination of the ring of Siegel modular forms depends on the determination of the kernel of $R_{g} \rightarrow A_{g}$. Along these lines the cases $g \leq 3$ have been treated successfully (reproducing and enlarging known results) [Ru1], [Ru2].

This paper is an approach to attack the case $g=4$. What we get is a simple element of the kernel. The second author determined the dimension 
formula for the ring $R_{4}[\mathrm{Ou}]$. The dimension of the space of Siegel modular forms in genus four is known in some cases:

$\begin{array}{lccccccc}\text { weight } k & 4 & 6 & 8 & 10 & 12 & 14 & 16 \\ \operatorname{dim}\left(R_{4}\right)_{k} & 1 & 1 & 2 & 3 & 7 & 7 & 19 \\ \operatorname{dim}\left[\Gamma_{4}, k\right] & 1 & 1 & 2 & ? & 6 & ? & ?\end{array}$

This table shows that there must be a relation in weight 12 , i.e. there must exist an invariant polynomial of degree 24 in the 16 variables $F_{a}$ which is not identically zero but which vanishes if one replaces $F_{a} \mapsto f_{a}$. We use codes to determine this polynomial. Recall that a (binary) code $C$ is nothing else but a subvector space $C \subset \mathbb{F}_{2}^{n}$. The code ploynomial of genus $g$ associated to such a code is

$$
P(C)=P^{(g)}(C):=\sum_{\alpha_{1}, \ldots, \alpha_{g} \in C} \prod_{a \in \mathbb{F}_{2}^{g}} F_{a}^{a\left(\alpha_{1}, \ldots, \alpha_{g}\right)},
$$

where $a\left(\alpha_{1}, \ldots, \alpha_{g}\right)$ denotes the number of all $i \in\{1, \ldots, n\}$ such that $a=\left(\alpha_{1 i}, \ldots, \alpha_{g i}\right)$. The weight of an element $\alpha \in C$ is the number of digits 1 in $\alpha$. A code is called doubly-even if all weights are divisible by four. A code is called self-dual if it agrees with its orthogonal complement with respect to $a \cdot b$.

It is well-known [Du], [Gl], [Ru1], that a $H_{g}$-invariant polynomial $P \in$ $R_{g}$ is a linear combination of code polynomials of self-dual doubly-even codes if and only if its degree is divisible by 8 . In our case $(g=4$ and $k=12$ ) we have to consider codes in $\mathbb{F}_{2}^{24}$. It is known that up to isomorphism precisely 9 self-dual doubly-even codes exist. We denote representatives by $C_{1}, \ldots, C_{9}$. The first seven denote the indecomposable ones. We refer to the list [PS]. The 9 types are characterized by their root subcodes which are direct products of the codes $a_{n}, d_{n}, n \geq 4, e_{7}, e_{8}$. The correspondence is as follows:

$$
\begin{array}{ccccccccc}
C_{1} & C_{2} & C_{3} & C_{4} & C_{5} & C_{6} & C_{7} & C_{8} & C_{9} \\
d_{12}^{2} & d_{10} e_{7}^{2} & d_{8}^{3} & d_{6}^{4} & d_{24} & d_{4}^{6} & a_{1}^{24} & d_{16} e_{8} & e_{8}^{3}
\end{array}
$$

First we consider the 9 Siegel modular forms. We know that the dimension of the space $\left[\Gamma_{4}, 12\right]$ is 6 .

Proposition 1.1. The Siegel modular forms $S_{i}=S\left(C_{i}\right)$ of genus 4 which are attached to the nine codes $C_{i}$ generate the six dimensional space 
$\left[\Gamma_{4}, 12\right]$. A basis is given by $S_{1}, S_{2}, S_{3}, S_{4}, S_{6}, S_{7}$. The expressions of the three remaining terms with respect to this basis is

$$
\begin{aligned}
S_{5} & =66 S_{1}-495 S_{3}+880 S_{4}-594 S_{6}+144 S_{7}, \\
3 S_{8} & =17 S_{1}+20 S_{2}-145 S_{3}+208 S_{4}-125 S_{6}+28 S_{7}, \\
S_{9} & =3 S_{1}+20 S_{2}-75 S_{3}+96 S_{4}-55 S_{6}+12 S_{7} .
\end{aligned}
$$

Each of the codes $C_{i}$ corresponds to one of the 24 Niemeier lattices and the modular form $S_{i}$ is the theta series of this Niemeier lattice. The proof of Propisition 1.1 uses the knowledge of some Fourier coefficients. Recall that the theta series $\vartheta(L ; Z)$ of genus $g$ of a definite lattice $L$ admits a Fourier expansion whose Fourier coefficients are given by the number of isometric embeddings of an arbitrary lattice $M$ of rank $\leq g$ into $L$. In case of the 24 Niemeier lattices $L$ and the irreducible root lattices $M$ of rank $\leq 12$ these numbers can be found in $[\mathrm{BFW}]$. For our purpose it is sufficient to take the six root lattices $A_{0}, A_{1}, A_{2}, A_{3}, A_{4}, D_{4}$ (and the 9 Niemeier code lattices) to seperate the theta series. In [BFW] they carry the same name as the corresponding codes but using capital letters. For example the Neimeier lattice corresponding to the code $e_{8}$ is the lattice $E_{8}$.

As we know from the results $[\mathrm{Ou}]$ the space generated by the code polynomials has dimension 7 . To get a basis we computed the coefficients of the monomial

$$
F_{0}^{9} \prod_{a \neq 0} F_{a}
$$

in the code polynomial $P_{i}=P\left(C_{i}\right)$. This monomial (to be precise an equivalent one) carries number 127 in our tables.

Proposition 1.2. The coefficients corresponding to the monomial $F_{0}^{9} \prod_{a \neq 0} F_{a}$ of the nine code polynomials $P_{i}=P\left(C_{i}\right)$ are

$$
0, \quad 70, \quad 24, \quad 72, \quad 0, \quad 148, \quad 253, \quad 80,168
$$

multiplied with $2^{10} 3^{3} 5 \cdot 7$.

From Propositions 1.1 and 1.2 we obtain

TheOREM 1.3. The code polynomial

$$
R:=3 P_{1}+20 P_{2}-75 P_{3}+96 P_{4}-55 P_{6}+12 P_{7}-P_{9}
$$

does not vanish but the corresponding Siegel modular form is zero. 
We can consider the same linear combination of code polynomials in genus 5. Computing one further Fourier coefficient one sees that the corresponding Siegel modular form is not 0 .

THEOREM 1.4. The linear combination $R$ considered in genus five defines a non-vanishing Siegel cusp form of weight 12 and genus 5 with respect to the full Siegel modular group.

For sake of completeness we give the relations for the 9 code polynomials in genus 4:

Proposition 1.5. A basis for the $\mathrm{H}_{4}$-invariant code polynomials is given by $P_{1}, P_{2}, P_{3}, P_{4}, P_{6}, P_{7}, P_{8}$. The expressions for the two remaining are

$$
\begin{aligned}
& P_{5}=66 P_{1}-495 P_{3}+880 P_{4}-594 P_{6}+144 P_{7} \\
& P_{9}=-14 P_{1}+70 P_{3}-112 P_{4}+70 P_{6}-16 P_{7}+3 P_{8} .
\end{aligned}
$$

There is a homomorphism

$$
\Phi: R_{g} \longrightarrow R_{g-1}, \quad F_{\left(a_{1}, a_{2}, a_{3}, a_{4}\right)} \longmapsto \begin{cases}0 & \text { if } a_{4}=0 \\ F_{\left(a_{1}, a_{2}, a_{3}\right)} & \text { otherwise. }\end{cases}
$$

This induces the Siegel $\Phi$-operator on the level of modular forms. We apply this operator to the relation $R$ (see Theorem 1.3). We get a relation in genus three. In this case there is a defining relation of degree 16 [Ru1]. It is the code polynomial

$$
P^{(3)}\left(e_{8}^{2}\right)-P^{(3)}\left(d_{16}\right)
$$

The upper index 3 indicates that we consider code polynomials of genus three. Recall that we denote by $e_{8}$ resp. $d_{16}$ the unique irreducible self-dual doubly-even code in dimension 8 resp. 16. So $R \mid \Phi$ must be a product of this polynomial and an invariant polynomial of degree 8 . There is only one up to a constant factor, namely $P^{(3)}\left(e_{8}\right)$. We obtain

Proposition 1.6. The image of the relation $R$ (see Theorem 1.3) under the $\Phi$-operator is

$$
R \mid \Phi=\left(P^{(3)}\left(e_{8}^{2}\right)-P^{(3)}\left(d_{16}\right)\right) \cdot P^{(3)}\left(e_{8}\right) .
$$


Only the constant has to be verified. To do this we use the coefficients of the monomial

$$
F_{(0,0,0,0)}^{16} \prod_{a_{4}=0} F_{a}
$$

An equivalent one carries number 40 in our table. The coefficients of the nine $C_{i}$ in our ordering are

$$
0, \quad 336, \quad 0, \quad 0, \quad 0, \quad 0, \quad 0, \quad 1344,4032 .
$$

Hence the coefficient of $R$ is 2688 . This is also the coefficient of $R \mid \Phi$ with respect to the degree-3-monomial

$$
F_{(0,0,0)}^{16} \prod_{a \in \mathbb{F}_{2}^{3}} F_{a}
$$

On the other hand the coefficient of $\left(P^{(3)}\left(e_{8}^{2}\right)-P^{(3)}\left(d_{16}\right)\right) \cdot P^{(3)}\left(e_{8}\right)$ is also 2688 (see [Ru3]).

\section{$\S 2$. Admissible monomials}

The number of monomials of degree 24 in 16 variables is 25140840660 . But not all of them can occur in a code polynomial of a self-dual doublyeven code.

Definition 2.1. A monomial $\left(\alpha_{a}\right)$ is called admissible if

$$
\sum_{a \in \mathbb{F}_{2}^{g}} \alpha_{a} \equiv 0 \bmod 8 \text { and } \sum_{a \in \mathbb{F}_{2}^{g}} \alpha_{a} S[a] \equiv 0 \bmod 4
$$

for all integral symmetric $g \times g$-matrices $S$.

Here we use the usual notation $S[a]=\sum_{i, j} s_{i j} a_{i} a_{j}$. It is of course sufficient to take for $S$ a system of generators, for example all elements in the diagonal or above the diagonal are zero besides one which is 1 . It is easy to prove (see [Ru3]) that in the code polynomial of self-dual doubly-even codes only admissible monomials appear.

There is a certain group $\operatorname{AGL}(g)$ which acts on the set of admissible monomials, namely the semidirect product

$$
\operatorname{AGL}(g)=\operatorname{GL}\left(g, \mathbb{F}_{2}\right) \cdot \mathbb{F}_{2}^{g}
$$


The group $\operatorname{AGL}(g)$ acts on $\mathbb{F}_{2}^{g}$ as the group of affine transformations,

$$
x \longmapsto U x+a, \quad(U, a) \in \operatorname{AGL}(g) .
$$

The order of AGL(4) is

$$
\sharp \operatorname{AGL}(4)=2^{4}\left(2^{4}-1\right)\left(2^{4}-2\right)\left(2^{4}-2^{2}\right)\left(2^{4}-2^{3}\right)=322560=2^{10} 3^{2} 5 \cdot 7 .
$$

The group $\operatorname{AGL}(g)$ is a subgroup of $H_{g}$. Hence our code polynomials are invariant under $\operatorname{AGL}(g)$. Our goal is to determine a complete system of representatives of admissible monomials of weight $w t(a)=\sum_{a} \alpha_{a}=24$.

Therefore it is sufficient to consider a system of representatives of all admissible monomials under $\operatorname{AGL}(g)$. We determined a system of representatives in the case $g=4$.

Proposition 2.2. There exist 160 admissible monomials of weight 24, up to $\mathrm{AGL}(4)$.

An explicit list is given in Table I below.

We give some explanations how this table has been computed. For the computations we used the computer algebra system MAGMA. The admissible monomials can be considered as elements of $\mathbb{Z}^{16}$. The group AGL(4) acts on this domain permuting the components. A monomial $\left(\alpha_{a}\right) \in$ $\mathbb{Z}^{16}$ is called admissible $\bmod N$, if

$$
\sum_{a \in \mathbb{F}_{2}^{g}} \alpha_{a} \equiv 0 \bmod (8, N) \text { and } \sum_{a \in \mathbb{F}_{2}^{g}} \alpha_{a} S[a] \equiv 0 \bmod (4, N)
$$

for all integral symmetric $g \times g$-matrices $S$. This condition depends only on $\alpha \bmod \mathrm{N}$, hence this condition can be defined for elements $\alpha \in(\mathbb{Z} / N \mathbb{Z})^{16}$. Let $\beta \in \mathbb{Z}^{16}$ be the reduced representative $\bmod N$ of $\alpha$, i.e. $0 \leq \beta_{i} \leq N$ for all $i$. We use the notation

$$
w t(\alpha, N):=\sum_{i=1}^{16} \beta_{i}
$$

We consider the set

$$
\mathcal{A}(N):=\left\{\alpha \in(\mathbb{Z} / N \mathbb{Z})^{16} ; w t(\alpha, N) \leq 24\right\}
$$


The idea is to compute step by step a system of representatives of the sets $\mathcal{A}(2), \mathcal{A}(4), \mathcal{A}(8), \mathcal{A}(16)$.

The first step is to determine the admissible monomials mod 2. The number of all possibilities $2^{16}=65536$ is small enough to compute by a silly computer program. We have 3 representatives. In the next step one considers, for each $\alpha \in(\mathbb{Z} / 2 \mathbb{Z})^{16}$ of the three, the possible expansions $\beta \in(\mathbb{Z} / 4 \mathbb{Z})^{16}$ to admissibles $\bmod 4$ with the property $w t(\beta, 4) \leq 24$. We take a system of representatives under the stabilizer of $\alpha$ in AGL(4). Using this method we found that $\mathcal{A}(4)$ has 22 representatives and similarly that $\mathcal{A}(8), \mathcal{A}(16)$ have 109,156 representatives respectively. In the last step one expands to $\mathcal{A}(24)$ which gives the final result.

The computation of the coefficients of the monomials is more involved. For each member $C$ of the nine codes one needs a system of representatives $\left(\alpha_{1}, \alpha_{2}, \alpha_{3}, \alpha_{4}\right)$ of code words with respect to the action of the automorphism group of $C$. One restricts to tuples such that the corresponding monomial belongs to the list of the 160 representatives. One starts with a list of representatives of code words $\alpha_{1}$, computes the stabilizer of $\alpha_{1}$ and determines a system of representatives $\alpha_{2}$ with respect to this stabilizer, and so on. Again we used the MAGMA system which provides programs to handle codes and their automorphism groups. The result of the computations is in Table II.

The programs and tables can be found on the WEB-page http://www.rzusers.uni-heidelberg.de/ ${ }^{\sim}$ t91

We would like to mention one further result of the calculations:

Remark 2.3. The total number of monomials with non-zero coefficients in the code polynomial $R$ (see Theorem 1.3) is 2004480 .

\section{$\S 3$. Tables}

The following table gives a system of representatives of admissible monomials. The tuple $\left(\alpha_{1}, \ldots, \alpha_{16}\right)$ stands for the monomial $\prod F_{i}^{\alpha_{i}}$. We use the binary convention, i.e. the characteristic $a=\left(a_{1}, a_{2}, a_{3}, a_{4}\right)$ is encoded as digit $i=8 a_{1}+4 a_{2}+2 a_{3}+a_{4}$. The number behind the monomial gives the length of the orbit of the monomial with respect to the group $\operatorname{AGL}(4)$. 
Table I

$1(0,0,0,2,0,0,0,2,0,6,4,0,4,6,0,0) \quad 40320$

$2(1,2,0,1,6,1,1,0,1,2,0,1,6,1,1,0) \quad 2520$

$3(0,8,0,2,0,0,0,2,0,2,0,0,0,6,0,4) \quad 20160$

$4(0,0,0,2,8,0,0,10,0,2,0,0,0,2,0,0) 6720$

$5(0,0,0,0,2,0,2,0,2,14,0,0,2,0,0,2) 2688$

$6(1,0,0,1,4,1,1,4,3,0,0,3,0,3,3,0) \quad 5040$

$7(1,0,2,3,0,1,3,2,3,2,0,1,2,3,1,0) \quad 840$

$8(0,2,0,2,4,2,2,4,2,0,2,0,0,2,2,0) \quad 20160$

$9(0,0,0,2,0,0,0,10,0,6,0,0,0,6,0,0) 1680$

$10(0,0,0,0,2,0,2,4,2,2,4,0,2,4,0,2) 26880$

$11(1,0,0,3,0,1,1,2,1,2,0,1,0,11,1,0) 6720$

$12(1,0,0,1,0,1,1,0,11,0,0,3,0,3,3,0) 1680$

$13(3,0,2,1,0,1,1,0,1,2,0,3,2,3,3,2) \quad 13440$

$14(1,2,0,1,2,1,1,4,1,2,4,1,2,1,1,0) \quad 2520$

$15(3,0,0,1,0,7,1,0,1,2,0,1,0,3,3,2) \quad 26880$

$16(0,0,2,2,0,0,2,2,2,2,0,4,2,2,4,0) \quad 840$

$17(1,0,4,1,0,1,5,4,1,4,0,1,0,1,1,0) \quad 13440$

$18(1,4,0,1,0,1,1,0,1,4,4,1,4,1,1,0) \quad 1680$

$19(3,2,0,1,0,1,3,2,3,0,2,1,2,1,3,0) \quad 5040$

$20(1,0,0,1,0,9,1,0,3,0,0,3,0,3,3,0) \quad 1680$

$21(5,0,0,3,0,1,1,2,5,2,0,1,0,3,1,0) \quad 40320$

$22(1,0,0,1,6,1,3,0,3,2,0,1,2,1,1,2) \quad 26880$

$23(0,0,0,2,0,0,0,2,0,2,0,0,0,18,0,0) 560$

$24(3,0,0,1,0,3,1,0,5,2,0,1,0,3,3,2) \quad 26880$

$25(2,2,2,0,2,2,0,2,2,2,0,2,0,4,0,2) \quad 2688$

$26(1,0,0,3,4,1,1,6,1,2,0,1,0,3,1,0) \quad 40320$

$27(0,2,0,2,0,2,2,4,2,0,2,0,4,2,2,0) \quad 3360$

$28(0,0,0,0,0,0,0,0,24,0,0,0,0,0,0,0) 16$

$29(0,0,2,2,8,0,2,2,2,2,0,0,2,2,0,0) \quad 240$

$30(1,0,0,3,0,1,1,2,9,2,0,1,0,3,1,0) \quad 20160$

$31(1,0,0,5,4,1,1,0,9,0,0,1,0,1,1,0) \quad 13440$

$32(1,0,0,5,0,5,1,0,3,0,0,3,0,3,3,0) \quad 2520$

$33(5,6,0,1,2,1,1,0,1,2,0,1,2,1,1,0) \quad 13440$

$34(0,4,0,2,0,0,0,2,8,2,0,0,0,2,0,4) \quad 20160$

$35(1,2,0,1,10,1,1,0,1,2,0,1,2,1,1,0) 1680$

$36(0,2,0,2,0,2,2,0,2,0,2,4,0,6,2,0) \quad 40320$

$37(0,0,0,2,4,0,0,2,4,2,0,0,4,2,4,0) \quad 10080$

$38(1,0,0,1,8,1,1,0,1,0,0,5,0,1,5,0) \quad 6720$

$39(0,0,2,2,4,0,2,6,2,2,0,0,2,2,0,0) \quad 1920$

$40(1,0,0,1,0,1,1,0,1,0,0,1,0,1,17,0) 240$

$41(0,4,0,2,0,8,0,2,0,2,0,0,0,2,0,4) \quad 5040$

$42(1,4,0,1,0,1,1,0,3,0,0,3,0,3,3,4) \quad 6720$

$43(1,1,3,3,1,1,1,1,1,1,1,1,1,1,3,3) \quad 140$

$44(1,0,0,1,8,1,1,0,3,0,0,3,0,3,3,0) \quad 3360$

$45(0,6,0,2,0,2,2,0,2,0,2,0,0,2,6,0) 20160$

$46(1,0,0,1,8,1,1,8,1,0,0,1,0,1,1,0) \quad 840$

$47(0,0,0,2,0,0,0,2,8,6,0,0,0,6,0,0) \quad 10080$

$48(1,4,0,1,2,1,3,0,3,2,0,1,2,1,1,2) \quad 26880$

$49(1,2,4,5,2,1,1,0,1,2,0,1,2,1,1,0) \quad 13440$

$50(1,0,0,3,0,1,1,6,1,2,0,1,0,3,5,0) \quad 40320$
$51(0,0,0,2,0,4,0,6,0,2,0,0,4,2,0,4) \quad 80640$

$52(1,4,0,1,8,1,1,0,1,0,0,1,0,1,1,4) \quad 5040$

$53(3,0,0,1,0,3,1,0,1,6,0,1,0,3,3,2) \quad 13440$

$54(1,4,0,3,0,1,1,2,1,2,0,1,0,3,1,4) \quad 10080$

$55(0,0,4,0,4,0,0,0,8,0,0,0,4,4,0,0) \quad 13440$

$56(1,0,8,9,0,1,1,0,1,0,0,1,0,1,1,0) \quad 1920$

$57 \quad(0,0,8,0,12,0,0,4,0,0,0,0,0,0,0,0) \quad 3360$

$58(1,0,0,7,0,1,1,2,1,2,0,1,0,7,1,0) \quad 3360$

$59(1,0,2,1,2,3,1,2,1,2,0,1,2,1,3,2) \quad 3360$

$60(0,0,0,0,0,0,0,0,8,0,0,0,0,16,0,0) \quad 240$

$61(0,4,0,2,0,0,0,6,0,6,0,0,0,6,0,0) \quad 6720$

$62(0,0,0,0,4,0,0,4,0,0,0,0,0,0,16,0) \quad 1680$

$63(0,0,0,2,0,0,0,2,0,2,0,0,0,14,0,4) \quad 6720$

$64(0,0,2,2,0,0,6,2,6,2,0,0,2,2,0,0) \quad 840$

$65(0,4,0,6,0,4,0,2,0,2,0,0,0,2,0,4) \quad 6720$

$66(0,0,4,2,0,0,4,2,4,2,4,0,0,2,0,0) \quad 13440$

$67(0,0,0,10,0,0,0,10,0,2,0,0,0,2,0,0) 840$

$68(9,0,0,1,0,1,1,0,9,0,0,1,0,1,1,0) \quad 840$

$69(0,0,0,2,0,0,4,6,0,2,0,0,4,6,0,0) \quad 5040$

$70(4,0,4,0,2,0,2,0,2,2,0,4,2,0,0,2) \quad 26880$

$71 \quad(0,4,4,2,0,0,0,2,8,2,0,0,0,2,0,0) \quad 40320$

$72(0,4,0,0,6,0,6,0,2,2,0,0,2,0,0,2) \quad 40320$

$73(1,2,0,1,6,1,1,4,1,2,0,1,2,1,1,0) \quad 6720$

$74(1,0,0,1,0,1,1,0,3,0,0,7,0,7,3,0) \quad 2520$

$75(1,2,0,1,2,1,1,0,9,2,0,1,2,1,1,0) \quad 3360$

$76 \quad(0,4,4,2,0,0,0,2,0,2,0,0,0,10,0,0) \quad 26880$

$77 \quad(1,4,0,1,4,1,1,0,1,4,0,1,4,1,1,0) \quad 420$

$78(1,0,0,1,0,1,1,0,9,0,0,5,0,1,5,0) \quad 5040$

$79 \quad(0,2,2,2,0,2,2,2,2,2,0,2,2,2,0,2) \quad 140$

$80(0,0,0,2,0,0,0,2,8,2,0,0,0,6,0,4) \quad 53760$

$81 \quad(0,0,8,8,4,0,0,4,0,0,0,0,0,0,0,0) \quad 10080$

$82(1,0,0,1,0,1,5,0,7,0,0,3,0,3,3,0) \quad 6720$

$83(0,0,0,0,12,0,0,12,0,0,0,0,0,0,0,0) 120$

$84(1,0,0,1,2,1,3,0,7,2,0,1,2,1,1,2) \quad 13440$

$85(4,0,0,0,2,4,2,0,2,6,0,0,2,0,0,2) \quad 40320$

$86(1,0,0,13,4,1,1,0,1,0,0,1,0,1,1,0) \quad 1920$

$87(0,2,0,2,8,2,2,0,2,0,2,0,0,2,2,0) \quad 6720$

$88(4,0,0,0,0,4,0,0,0,4,0,0,0,4,4,4) \quad 448$

$89(0,0,4,4,0,4,0,0,0,0,4,0,0,0,4,4) \quad 6720$

$90(0,0,4,0,12,0,0,0,0,0,0,0,4,4,0,0) \quad 6720$

$91 \quad(1,0,0,3,0,1,1,2,1,2,0,5,0,7,1,0) \quad 40320$

$92(4,4,0,2,0,0,4,6,0,2,0,0,0,2,0,0) \quad 8960$

$93(1,8,0,5,4,1,1,0,1,0,0,1,0,1,1,0) \quad 13440$

$94(3,2,2,3,0,3,3,0,3,0,0,3,0,1,1,0) \quad 3360$

$95(1,4,0,1,0,1,1,0,9,0,0,1,0,1,1,4) \quad 6720$

$96(1,0,0,1,0,1,1,0,1,0,0,1,0,1,1,16) \quad 240$

$97(0,4,0,2,0,0,0,2,0,10,0,0,0,2,0,4) \quad 10080$

$98(0,0,0,2,0,0,0,2,8,2,0,0,0,2,8,0) \quad 6720$

$99(0,0,0,2,0,0,0,2,0,2,0,0,0,6,0,12) \quad 6720$

$100(1,0,0,3,0,1,5,2,5,2,0,1,0,3,1,0) \quad 10080$ 
$101(0,4,4,2,8,0,0,2,0,2,0,0,0,2,0,0) 26880$ $102(0,0,0,0,2,0,2,0,10,6,0,0,2,0,0,2) 13440$ $103(1,0,0,1,0,1,1,0,1,0,0,13,0,1,5,0) 1680$ $104(1,0,0,3,0,1,1,2,5,2,0,1,4,3,1,0) 40320$ $105(0,0,0,0,2,8,2,0,2,2,0,4,2,0,0,2) 40320$ $106(4,4,0,0,0,0,12,4,0,0,0,0,0,0,0,0) 560$ $107(3,0,0,3,0,3,3,0,3,0,0,3,0,3,3,0) \quad 30$ $108(0,0,4,2,0,0,4,2,4,2,0,0,4,2,0,0) 420$ $109(1,0,0,3,0,1,1,2,1,10,0,1,0,3,1,0) 6720$ $110(0,0,0,2,0,0,0,2,0,14,0,0,0,6,0,0) 1680$ $111(2,2,2,0,2,2,0,2,6,2,0,2,0,0,0,2) 4480$ $112(1,0,4,5,0,1,5,0,1,0,0,1,0,5,1,0) 13440$ $113(0,4,0,0,6,0,2,0,2,2,0,0,2,0,4,2) \quad 80640$ $114(0,4,0,2,0,0,0,2,0,6,4,0,4,2,0,0) 26880$ $115(4,0,0,2,0,0,0,2,0,2,0,0,4,2,4,4) \quad 40320$ $116(0,12,0,2,0,0,0,2,0,2,0,0,0,2,0,4) 5040$ $117(1,0,0,7,0,1,1,6,1,2,0,1,0,3,1,0) \quad 13440$ $118(1,0,4,1,0,1,1,0,7,0,0,3,0,3,3,0) \quad 13440$ $119(0,6,0,2,0,2,2,0,2,0,6,0,0,2,2,0) 3360$ $120(3,0,0,1,4,3,1,0,1,2,0,1,0,3,3,2) \quad 40320$ $121(1,0,0,3,0,1,1,6,1,6,0,1,0,3,1,0) \quad 3360$ $122(0,2,4,6,0,2,2,0,2,0,2,0,0,2,2,0) \quad 13440$ $123(0,0,4,2,0,0,4,6,0,6,0,0,0,2,0,0) \quad 10080$ $124(1,2,2,1,2,1,1,2,1,2,2,1,2,1,1,2) \quad 30$ $125(8,0,0,0,0,8,0,0,0,8,0,0,0,0,0,0) 560$ $126(4,4,0,0,0,0,4,4,8,0,0,0,0,0,0,0) \quad 1680$ $127(1,1,1,1,1,1,1,1,9,1,1,1,1,1,1,1) \quad 16$ $128(0,0,4,0,4,0,0,0,0,0,8,0,4,4,0,0) \quad 6720$ $129(0,0,0,0,2,0,2,0,10,2,0,4,2,0,0,2) 26880$ $130(1,0,0,3,0,1,1,2,1,2,4,1,0,3,1,4) 40320$
$131(0,2,0,2,0,2,2,0,10,0,2,0,0,2,2,0) 6720$ $132(0,0,0,0,2,0,2,0,2,6,0,8,2,0,0,2) 26880$ $133(0,4,4,0,4,0,0,4,0,0,0,0,4,0,0,4) \quad 840$ $134(0,0,0,0,6,0,2,0,2,2,0,0,6,0,0,6) \quad 8960$ $135(1,2,0,1,2,1,1,0,1,2,0,5,2,5,1,0) \quad 6720$ $136(1,1,1,1,5,1,1,5,1,1,1,1,1,1,1,1) \quad 120$ $137(0,0,0,0,4,0,0,20,0,0,0,0,0,0,0,0) 240$ $138(5,4,0,3,0,1,1,2,1,2,0,1,0,3,1,0) \quad 80640$ $139(1,0,0,3,8,1,1,2,1,2,0,1,0,3,1,0) 20160$ $140(4,0,0,0,2,0,2,0,6,2,0,0,6,0,0,2) 26880$ $141(1,0,0,1,2,1,3,0,3,2,0,5,2,1,1,2) 40320$ $142(1,0,0,5,12,1,1,0,1,0,0,1,0,1,1,0) 1920$ $143(4,4,0,2,0,0,0,2,0,2,0,0,0,2,4,4) 5040$ $144(1,0,4,5,0,1,1,0,3,0,0,3,0,3,3,0) \quad 13440$ $145(0,0,0,2,0,0,0,2,0,10,0,0,0,6,0,4) 20160$ $146(5,0,0,5,0,5,5,0,1,0,0,1,0,1,1,0) 420$ $147(1,2,0,1,2,1,1,0,1,2,8,1,2,1,1,0) \quad 1680$ $148(8,0,0,8,4,0,0,4,0,0,0,0,0,0,0,0) \quad 840$ $149(5,4,0,1,0,1,5,4,1,0,0,1,0,1,1,0) \quad 3360$ $150(0,0,0,6,0,0,0,6,0,6,0,0,0,6,0,0) \quad 140$ $151(0,0,2,2,0,0,2,2,10,2,0,0,2,2,0,0) 240$ $152(0,0,0,2,0,0,8,2,8,2,0,0,0,2,0,0) 2520$ $153(0,12,4,2,0,0,0,2,0,2,0,0,0,2,0,0) 13440$ $154(1,0,0,5,0,5,5,0,1,0,0,1,0,5,1,0) \quad 1680$ $155(1,12,0,1,0,1,1,0,1,0,0,1,0,1,1,4) 1680$ $156(5,0,0,1,0,1,1,0,1,0,0,1,4,1,5,4) 20160$ $157(0,0,0,2,0,0,0,2,0,2,16,0,0,2,0,0) 1680$ $158(0,0,0,0,2,0,2,0,2,2,0,12,2,0,0,2) 4480$ $159(1,0,4,7,0,1,1,2,1,2,0,1,0,3,1,0) \quad 40320$ $160(1,2,0,5,2,1,1,0,1,2,0,5,2,1,1,0) \quad 5040$

The following table gives the coefficients of the 9 Niemeier codes. The number in the first column refers to the 160 monomials as in Table I. The second line is the greatest common divisor of the coefficients of the 9 Niemeier codes. The last 9 columns give the coefficients divided by the greatest common divisor. For example the coefficient of the 127-th monomial $(1,1,1,1,1,1,1,1,9,1,1,1,1,1,1,1)$ (i.e. $\left.F_{(1,0,0,0)}^{8} \prod F_{a}\right)$ with respect to the code $C_{7}$ (this is the so-called Golay code) is given by $967680 \cdot 253$.

\section{Table II}

$\begin{array}{lllllllllll}\text { MON GCD } & C_{1} & C_{2} & C_{3} & C_{4} & C_{5} & C_{6} & C_{7} & C_{8} & C_{9} \\ 1 & 96 & 3720 & 1715 & 882 & 297 & 34650 & 60 & 0 & 8526 & 6174 \\ 2 & 9216 & 12300 & 7301 & 4440 & 2064 & 73920 & 600 & 0 & 23030 & 18522 \\ 3 & 72 & 27660 & 24143 & 18996 & 13335 & 23100 & 6960 & 0 & 33236 & 35868 \\ 4 & 24 & 1560 & 1057 & 498 & 171 & 6930 & 0 & 0 & 3430 & 4158 \\ 5 & 120 & 12 & 1 & 0 & 0 & 792 & 0 & 0 & 56 & 0 \\ 6 & 768 & 186000 & 133525 & 100800 & 70836 & 1478400 & 39120 & 0 & 3547600 & 321048 \\ 7 & 27648 & 232000 & 172725 & 117600 & 71028 & 739200 & 31760 & 0 & 378280 & 386904\end{array}$




\begin{tabular}{|c|c|c|c|c|c|c|c|c|c|}
\hline MON GCD & $C_{1}$ & $C_{2}$ & $C_{3}$ & $C_{4}$ & $C_{5}$ & $C_{6}$ & $C_{7}$ & $C_{8}$ & $C_{9}$ \\
\hline 2304 & 78375 & 78400 & 88515 & 99864 & 51975 & 115005 & 132825 & 75215 & 64827 \\
\hline 6 & 2652 & 2856 & 3047 & 3225 & 1155 & 3390 & 3542 & 2205 & 2205 \\
\hline 2160 & 8220 & 11515 & 13296 & 17010 & 27720 & 21856 & 28336 & 9800 & 16464 \\
\hline 192 & 1920 & 1561 & 576 & 180 & 0 & 0 & 0 & 4592 & 7056 \\
\hline 768 & 1080 & 1169 & 1008 & 810 & 0 & 480 & 0 & 1148 & 1764 \\
\hline 27648 & 51200 & 55125 & 60000 & 62292 & 0 & 63000 & 61985 & 33320 & 24696 \\
\hline 9216 & 410100 & 395675 & 401760 & 412938 & 1108800 & 430140 & 451605 & 473830 & 438354 \\
\hline 2304 & 6000 & 5355 & 5136 & 4140 & 0 & 2520 & 0 & 3920 & 0 \\
\hline 1152 & 339150 & 188650 & 182790 & 184356 & 23215 & 238290 & 345345 & 558110 & 228438 \\
\hline 288 & 120000 & 134995 & 117504 & 88848 & 0 & 47040 & & & 16464 \\
\hline 4608 & 7800 & 7350 & 16920 & 22608 & 0 & 26700 & 26565 & 4655 & 1029 \\
\hline 9216 & 400 & 184975 & 177600 & 180732 & 739200 & 192960 & 212520 & 288120 & 271656 \\
\hline 68 & 600 & 7805 & 9760 & 11898 & 0 & 14520 & 17710 & 3500 & 1764 \\
\hline 384 & & 791 & 708 & & 0 & 28800 & & 69776 & 49392 \\
\hline 304 & 2 & 39445 & 542 & 694 & 0 & 865 & 106260 & 392 & 0 \\
\hline & 1 & & 3 & & 55 & 0 & & 21 & 21 \\
\hline & 8800 & 54145 & 41232 & 28908 & 0 & 15000 & 0 & 480 & 98784 \\
\hline 5920 & 449 & 50225 & 540 & 56022 & 9240 & & & & 16464 \\
\hline & 156 & 132055 & 118 & 7444 & 0 & & & 520 & 49392 \\
\hline 115 & & 513275 & 373 & 240642 & 232 & 117 & & 107 & 1043406 \\
\hline & 1 & & 1 & 1 & 1 & 1 & 1 & 1 & 1 \\
\hline 4032 & 16 & 280 & 702 & 486 & 4950 & 1230 & 3795 & 1550 & 1638 \\
\hline & & 10045 & & 42 & 0 & 14 & & & 705 \\
\hline & 9 & 5929 & 23 & 57 & 0 & 0 & & & 35280 \\
\hline 1536 & & 160475 & 160 & 314 & 147840 & 159960 & 159390 & 64 & 160 \\
\hline 3456 & & 28175 & 227 & 352 & 0 & 9840 & 0 & & 49392 \\
\hline & & 2450 & 218 & & 519 & 45 & 0 & & 3969 \\
\hline 60 & & & 50 & 32 & 0 & 12 & & & 2646 \\
\hline & & & & 805 & 69300 & & 0 & & 61740 \\
\hline & & 208 & & 27846 & & 446 & 796 & & 27783 \\
\hline & & 3479 & 172 & & 0 & 0 & 0 & & \\
\hline & 660 & 815 & 57 & $45 !$ & 990 & 270 & & & 22932 \\
\hline & 0 & & 0 & 0 & 0 & 0 & 0 & 4 & 12 \\
\hline & & & & & & & & & 17787 \\
\hline & & & & & 0 & & & & \\
\hline 221184 & 00 & 1445 & & 232 & & & 115 & 199920 & 336 \\
\hline & & & & & 0 & & 0 & & \\
\hline & & & 21 & 10 & & 33 & & & 6174 \\
\hline & & & & 0 & & 0 & & & \\
\hline & & 2107 & 17 & & 11550 & 780 & 0 & & 4410 \\
\hline & & 1037575 & & 11 & 0 & 1140600 & & 120 & 889056 \\
\hline & & 496615 & & & 0 & & 850 & 680 & 148176 \\
\hline & & & & & 0 & & 0 & & \\
\hline & & & & & 207900 & & & & 61740 \\
\hline & & & & & 0 & & & & 2205 \\
\hline & & 2205 & 2792 & 3420 & 0 & & 8855 & 1960 & 0 \\
\hline & 350400 & 2750125 & 1992960 & 12891 & & 623520 & 0 & 5225360 & 53837 \\
\hline 72 & 82 & 245 & 117 & 27 & 17325 & 5 & 0 & & 1421 \\
\hline & 0 & 17 & 0 & 0 & & 0 & 0 & 72 & \\
\hline & & 47 & & & & 125 & & & \\
\hline & & 7831 & & 684 & 253440 & 0 & 0 & 50960 & 49392 \\
\hline 13824 & 1327200 & 1275225 & 1205760 & 1137588 & 1478400 & 106592 & 991760 & 1446480 & 1498224 \\
\hline 3 & 213 & 221 & 229 & 237 & 165 & 24 & 253 & & 197 \\
\hline 144 & & 3675 & 2988 & 2075 & & 1080 & 0 & 5292 & 4116 \\
\hline & & 68 & 39 & 18 & & 5 & 0 & & 203 \\
\hline 24 & & & 36 & 7 & & 0 & 0 & & 588 \\
\hline & & & & 39309 & & & & 37338 & 34986 \\
\hline 144 & 327900 & 354025 & 377820 & 397017 & 69300 & 412680 & 425040 & 257740 & 251076 \\
\hline
\end{tabular}




\begin{tabular}{|c|c|c|c|c|c|c|c|c|c|c|}
\hline MON & GCD & $C_{1}$ & $C_{2}$ & $C_{3}$ & $C_{4}$ & $C_{5}$ & $C_{6}$ & $C_{7}$ & $C_{8}$ & $C_{9}$ \\
\hline 66 & 288 & 49500 & 40425 & 36990 & 27378 & 103950 & 15060 & 0 & 47530 & 26754 \\
\hline 67 & 528 & 130 & 102 & 75 & 49 & 319 & 24 & 0 & 189 & 189 \\
\hline 68 & 96 & 4160 & 2309 & 1152 & 368 & 28160 & 0 & 0 & 9044 & 8316 \\
\hline 69 & 96 & 125040 & 96775 & 71082 & 46209 & 330330 & 22560 & 0 & 183358 & 179046 \\
\hline 70 & 432 & 235500 & 197225 & 157680 & 108774 & 138600 & 55680 & 0 & 252840 & 214032 \\
\hline 71 & 144 & 6600 & 3955 & 1818 & 648 & 34650 & 120 & 0 & 15750 & 17934 \\
\hline 72 & 48 & 13980 & 12691 & 7632 & 4374 & 138600 & 1440 & 0 & 33320 & 49392 \\
\hline 73 & 13824 & 7200 & 10780 & 13176 & 17226 & 0 & 21780 & 26565 & 6860 & 12348 \\
\hline 74 & 1536 & 9240 & 7379 & 5616 & 3786 & 21120 & 1920 & 0 & 12740 & 12348 \\
\hline 75 & 1152 & 3600 & 6125 & 5760 & 6048 & 0 & 4560 & 0 & 5320 & 10584 \\
\hline 76 & 48 & 900 & 455 & 126 & 27 & 20790 & 0 & 0 & 3738 & 4410 \\
\hline 77 & 4608 & 183000 & 110250 & 58320 & 24552 & 1108800 & 6240 & 0 & 404495 & 420861 \\
\hline 78 & 192 & 9840 & 8827 & 7392 & 5364 & 0 & 2880 & 0 & 9436 & 8820 \\
\hline 79 & 41472 & 301800 & 249900 & 224430 & 208632 & 1120350 & 203080 & 203665 & 457170 & 446586 \\
\hline 80 & 24 & 4260 & 2107 & 828 & 225 & 69300 & 0 & 0 & 11900 & 8820 \\
\hline 81 & 12 & 2835 & 1568 & 981 & 450 & 17325 & 135 & 0 & 5201 & 3633 \\
\hline 82 & 1536 & 15840 & 18137 & 20184 & 22302 & 0 & 24420 & 26565 & 11564 & 12348 \\
\hline 83 & 4 & 689 & 680 & 671 & 662 & 743 & 653 & 644 & 707 & 707 \\
\hline 84 & 4608 & 4200 & 6335 & 9576 & 14130 & 0 & 19860 & 26565 & 1960 & 0 \\
\hline 85 & 48 & 311100 & 229565 & 131328 & 62676 & 415800 & 17280 & 0 & 554680 & 691488 \\
\hline 86 & 336 & 0 & 17 & 0 & 0 & 0 & 0 & 0 & 112 & 336 \\
\hline 87 & 576 & 10950 & 5705 & 8298 & 6984 & 34650 & 4590 & 0 & 11410 & 882 \\
\hline 00 & 720 & 945 & 0 & 379 & 108 & 10395 & 361 & 1771 & & 343 \\
\hline 89 & 48 & 30375 & 12250 & 9405 & 4212 & 155925 & 1515 & 0 & 54145 & 29841 \\
\hline 90 & 24 & 345 & 175 & 81 & 27 & 3465 & 5 & 0 & 88 & 833 \\
\hline 91 & 384 & 32160 & 23653 & 14688 & 7308 & 0 & 2160 & 0 & 46256 & 49392 \\
\hline 92 & 2160 & 1620 & 1421 & 596 & 219 & 4620 & 0 & 0 & 4116 & 6860 \\
\hline 93 & 48 & 59520 & 33019 & 20736 & 7200 & 0 & 0 & 0 & 74480 & 35280 \\
\hline 9 & 4608 & 140000 & 99225 & 74880 & 47732 & 492800 & 23040 & 0 & 199920 & 148176 \\
\hline 95 & 480 & 1440 & 2107 & 960 & 432 & 0 & 0 & 0 & 1624 & 3528 \\
\hline 96 & 1344 & 0 & 0 & 0 & 0 & 0 & 0 & 0 & & 3 \\
\hline 97 & 48 & 11820 & 10045 & 8886 & 6687 & 207 & 3780 & 0 & 11914 & 7938 \\
\hline 98 & 48 & 1410 & 245 & 153 & 0 & & 0 & 0 & 3703 & 2079 \\
\hline 99 & 24 & 780 & 371 & 228 & 75 & 4620 & 0 & 0 & 1316 & 588 \\
\hline 100 & 384 & 307200 & 196735 & 108288 & 48636 & 1774080 & 12960 & 0 & 634256 & 642096 \\
\hline 101 & 480 & 2220 & 917 & 675 & 225 & 10395 & 0 & 0 & 3157 & 441 \\
\hline & 7 & 620 & 105 & 64 & 0 & 9240 & 0 & 0 & 1400 & 0 \\
\hline 103 & 576 & 80 & 63 & 32 & 12 & 0 & 0 & 0 & 140 & 196 \\
\hline 44 & 768 & 276000 & 238385 & 188352 & 130446 & 0 & 66960 & 0 & 307720 & 321048 \\
\hline 105 & 72 & 32700 & 26635 & 17424 & 9450 & 138600 & 2880 & 0 & 43400 & 35280 \\
\hline 6 & 24 & 5265 & 5831 & 6473 & 7191 & & & 8855 & & 4361 \\
\hline 107 & 43008 & 83600 & 77175 & 71280 & 65204 & 123200 & 59160 & 53130 & 95340 & 93492 \\
\hline 88 & 576 & 1745850 & 1669675 & 1592955 & 152015 & 42269575 & 1449660 & 1381380 & 1915165 & 1927317 \\
\hline 9 & 192 & 3840 & 3115 & 2112 & 900 & 0 & 0 & 0 & 4592 & 7056 \\
\hline & 288 & 40 & 34 & & 19 & 55 & 10 & 0 & & \\
\hline & 1728 & 16500 & 25725 & 39408 & 53076 & 46200 & 64720 & 70840 & 17640 & 32928 \\
\hline & 288 & 14400 & 11025 & & & 0 & & 0 & & 16464 \\
\hline & 48 & 102300 & 84035 & 79488 & 728 & 415800 & 40320 & 0 & 320 & 98784 \\
\hline & 144 & & & & & & & 0 & 14700 & 4116 \\
\hline & 192 & 68250 & 67375 & & 40302 & 155925 & 23490 & 0 & 110495 & 163611 \\
\hline & 96 & & 938 & 597 & 297 & 3465 & 90 & 0 & 2135 & 1911 \\
\hline & 768 & & 6629 & 31 & & 0 & 0 & 0 & 72 & 24696 \\
\hline & 384 & 400 & & 77 & 41 & 0 & 1200 & 0 & & 49392 \\
\hline & 3456 & 4150 & 1960 & 88 & 27 & 53130 & 50 & 0 & 11466 & 10290 \\
\hline 120 & 4608 & & & & & 0 & 68100 & 79695 & 37240 & 49392 \\
\hline & 384 & & & & & 591360 & 0 & & 126224 & 49392 \\
\hline 122 & 576 & 18600 & 16415 & 16524 & 23301 & 69300 & 35580 & 53130 & 28420 & 12348 \\
\hline & 96 & 27360 & 29743 & 32826 & 37809 & 34650 & 44520 & 53130 & 28126 & 30870 \\
\hline
\end{tabular}


E. FREITAG AND M. OURA

\begin{tabular}{|c|c|c|c|c|c|c|c|c|c|c|}
\hline $\mathrm{MON}$ & GCD & $C_{1}$ & $C_{2}$ & $C_{3}$ & $C_{4}$ & $C_{5}$ & $C_{6}$ & $C_{7}$ & $C_{8}$ & $C_{9}$ \\
\hline 124 & 232243 & 93000 & 80850 & 71880 & 62292 & 184800 & 53140 & 44275 & 112770 & 10260 \\
\hline & 18 & 845 & 833 & 869 & 953 & & 1085 & 1265 & 1013 & 1013 \\
\hline & 24 & 15075 & 10045 & 10455 & 12177 & 519 & 17355 & 26565 & 20335 & 7791 \\
\hline & 967680 & 0 & 70 & 24 & 72 & 0 & 148 & 253 & 80 & 168 \\
\hline & 120 & 6975 & 6097 & 4707 & 3321 & 10395 & 1755 & 0 & 9331 & 10731 \\
\hline & 24 & 9300 & 3745 & 1872 & 450 & 83160 & 0 & 0 & 18872 & 7056 \\
\hline 30 & 384 & 566400 & 750925 & 941760 & 109508 & 0 & 1209600 & 1275120 & 145040 & 49392 \\
\hline & 1728 & 470 & 210 & 126 & 45 & 2310 & 10 & 0 & 798 & 294 \\
\hline & 24 & 22020 & 14147 & 4896 & 1260 & 138600 & 0 & 0 & 68488 & 98784 \\
\hline & 144 & 175325 & 159250 & 156335 & & 544775 & 174545 & 194810 & 23495 & 223979 \\
\hline & 144 & 1060 & 245 & 288 & & 154 & & 0 & 1960 & 0 \\
\hline & 2304 & 30480 & 42287 & 48672 & 60840 & 0 & 78720 & 106260 & 32536 & 74088 \\
\hline 80 & 387072 & 6720 & 5082 & 5448 & 5928 & 21120 & 7100 & 8855 & 9072 & 5880 \\
\hline 77 & 6 & 5 & 4 & & 2 & 11 & 1 & 0 & 7 & 7 \\
\hline & 768 & 45600 & 61985 & 80352 & 103662 & 0 & 130320 & 15 ? & 25480 & 24696 \\
\hline & 192 & 33600 & 39655 & 41472 & 37332 & 0 & 24480 & 0 & 14000 & 7056 \\
\hline & & & 21805 & 11664 & 405 & 138600 & 0 & 0 & 644 & 49392 \\
\hline & 4608 & 61800 & 79625 & 93672 & 108414 & 0 & 121620 & 132825 & 37240 & 49392 \\
\hline & 336 & 0 & 51 & 0 & 0 & 0 & 0 & 0 & 112 & 336 \\
\hline & 192 & 282750 & 145775 & 86625 & 37386 & 2373525 & 10620 & 0 & 578935 & 398223 \\
\hline & 38 & 50400 & & 38592 & 28944 & 0 & 16320 & 0 & 50960 & 49392 \\
\hline & $7:$ & 146 & 102 & & & 462 & 80 & 0 & 2660 & 294 \\
\hline & & 800 & 49735 & 35136 & 22104 & 177408 & 10368 & 0 & 156 & 10 \\
\hline & 46( & 3 & & & & & & 26 & & 264 \\
\hline & & & & & & & & & & \\
\hline & 57 & 400 & 9735 & 23424 & 73 & 360 & 0 & 0 & 200312 & 205800 \\
\hline & & 25922 & 24990 & 24057 & 23123 & 31493 & & & & \\
\hline & & & & 570 & 741 & 330 & 97 & 12 & 386 & 546 \\
\hline & 14 & 8550 & 5329 & 2979 & 1332 & & 36 & 0 & 437 & 17157 \\
\hline & 48 & & 35 & 18 & 0 & 6930 & 0 & 0 & & 294 \\
\hline & 1152 & 12192 & 14455 & 16992 & 18936 & 0 & & & & 4116 \\
\hline & & & & & 0 & 0 & 0 & 0 & 329 & 147 \\
\hline & 57 & 13440 & 10535 & 10944 & 8712 & 0 & 5280 & 0 & 12152 & 8232 \\
\hline & 2 & & 5 & 3 & & 49 & 0 & 0 & & \\
\hline & & & 6 & 1 & 2 & 30 & & 0 & 616 & \\
\hline & & 20400 & 975 & 22896 & 18882 & 0 & 11160 & 0 & 13720 & 24696 \\
\hline & & & & & & 887040 & & 0 & & 17287 \\
\hline
\end{tabular}

\section{REFERENCES}

[BFW] R. Borcherds, E. Freitag, R. Weissauer, A Siegel cusp form of degree 12 and weight 12, J. reine angew. Math., 494 (1998), 141-153.

[Du] W. Duke, On codes and Siegel modular forms, Int. Math. Research Notoces (1993), 125-136.

[Gl] A. M. Gleason, Weight polynomials of self-dual codes and the MacWilliams identities, Actes Congrés Intern. des Mathématiciens (Nice 1970), Tome 3, Gauthier-Villars, Paris (1971), pp. 211-215.

[Ou] M. Oura, The dimension formula for the ring of code polynomials in genus 4, Osaka J. Math., 34 (1997), 53-72.

[PS] V. Pless and N. J. A. Sloane, On the classification and enumeration of self-dual codes, J. Comb. Th., A18 (1975), 313-335. 
[PY] C. Poor and D. Yuen, Dimensions of spaces of Siegel modular forms of low weight in degree four, Bull. Austral. Math. Soc., 54 (1996), 309-315.

[Ru1] B. Runge, On Siegel modular forms, Part I, J. reine angew. Math., 436 (1993), 57-85.

[Ru2] - On Siegel modular forms, Part II, Nagoya Math. J., 138 (1995), 179-197.

[Ru3] Codes and Siegel modular forms, Disc. Math., 148 (1996), 175-204.

Eberhard Freitag

Mathematisches Institut

Im Neuenheimer Feld 288, D69120

Heidelberg

freitag@mathi.uni-heidelberg.de

Manabu Oura

Graduate School of Mathematics

Kyushu University

Japan

ohura@math.kyushu-u.ac.jp 